\title{
A NOTE ON THE RELLICH FORMULA IN LIPSCHITZ DOMAINS
}

\author{
Alano Ancona
}

\begin{abstract}
Let $L$ be a symmetric second order uniformly elliptic operator in divergence form acting in a bounded Lipschitz domain $\Omega$ of $\mathbb{R}^{N}$ and having Lipschitz coefficients in $\Omega$. It is shown that the Rellich formula with respect to $\Omega$ and $L$ extends to all functions in the domain $\mathcal{D}=\left\{u \in H_{0}^{1}(\Omega) ; L(u) \in L^{2}(\Omega)\right\}$ of $L$. This answers a question of A. Chaïra and G. Lebeau.
\end{abstract}

\section{Introduction}

Let $L=\sum_{1 \leq i, j \leq N} \partial_{i}\left(a_{i j} \partial_{j .}\right)$ be a uniformly elliptic operator in divergence form in $\mathbb{R}^{N}$, the coefficients $a_{i j}$ being (real) Lipschitz continuous functions in $\mathbb{R}^{N}$ such that $a_{i j}=a_{j i}$ for $1 \leq i, j \leq N$. Let $\mathcal{A}$ denote the matrix $\left\{a_{i j}\right\}$.

If $\Omega \subset \mathbb{R}^{N}$ is a bounded Lipschitz domain in $\mathbb{R}^{N}$, if $V$ is a $C^{1}$ vector field in $\bar{\Omega}$ and if $u \in H^{2}(\Omega)$, then the following so-called Rellich formula holds (for references see Nečas [N, p. 224]).

$$
\begin{aligned}
& \text { (1) } \int_{\partial \Omega}\left\{\partial_{\nu_{L}}(u) \partial_{V}(u)-\frac{1}{2}\|\nabla u\|_{L}^{2}\langle V, \nu\rangle\right\} d \sigma \\
& =\int_{\Omega}\left\{d u(V) L(u)+d u\left(\partial_{\mathcal{A} \nabla u} V\right)-\frac{1}{2} \operatorname{div}(V)\|\nabla u\|_{L}^{2}-\frac{1}{2} q_{L, V}^{\prime}(\nabla u)\right\} d x
\end{aligned}
$$

where $\nu$ is the unit exterior normal field along $\partial \Omega$ and $\nu_{L}=\mathcal{A}(\nu)$ is the conormal field; $\partial_{U}$ denotes the differentiation operator in the direction $U$, and we have let $\|U\|_{L}^{2}=\langle\mathcal{A} U, U\rangle$ and $q_{L, V}^{\prime}(U)=\left\langle\partial_{V}(\mathcal{A})(U), U\right\rangle=$

Keywords. Rellich formula, Lipschitz domain, Harnack boundary principle.

1991 Mathematics subject classifications: 31C99, 35J15, 35L05. 
$\sum_{i, j} U_{i} U_{j} d a_{i j}(V)$ when $V \in \mathbb{R}^{N}$. At least if $u \in C^{2}(\bar{\Omega})$, the formula follows from the Stokes formula $\int_{\partial \Omega} W \cdot \nu d \sigma=\int_{\Omega} \operatorname{div}(W) d x$ on taking $W=d u(V) \mathcal{A}(\nabla u)-\frac{1}{2}\|\nabla u\|_{L}^{2} V$. The general case follows from an approximation argument. Of course the Lipschitz regularity of $\Omega$ is only needed in a neighborhood of $\operatorname{supp}(V) \cap \partial \Omega$.

In this note it is shown that the Rellich formula extends to all functions $u$ in the domain of $L$, that is $u \in H_{0}^{1}(\Omega)$ with $L(u) \in L^{2}(\Omega)$; this amounts $([\mathbf{N}])$ to a continuity property of the gradient of $L$-solutions with respect to perturbations of $\Omega$ (see Theorem 1 below). This extension of Rellich formula answers a question raised to me by A. Chaïra and G. Lebeau [CL] (see also [N, Problème 2.2, p. 258)] and is useful in some problems in control theory for the wave equation $([\mathbf{C}],[\mathbf{C L}])$. The proof relies on well-known results and methods of the Potential theory in Lipschitz domains (in particular $[\mathbf{D}],[\mathbf{A} \mathbf{1}],[\mathbf{J K} \mathbf{1}]$ and $[\mathbf{A 2}]$ ).

\section{Notations and preliminaries}

In this section we fix some notations and recall several basic properties of the Potential theory in Lipschitz domains with respect to elliptic second order operators.

2.1. Let $N$ be a fixed integer $\geq 2$ and let $\varphi: \mathbb{R}^{N-1} \rightarrow \mathbb{R}$ be a function such that $\varphi(0)=0$ and $|\varphi(x)-\varphi(y)| \leq k|x-y|$ for $x, y \in \mathbb{R}^{N-1}$ and a positive constant $k$. For $x \in \mathbb{R}^{N}$, we note $x=\left(x^{\prime}, x_{N}\right)$ the decomposition of $x$ in $\mathbb{R}^{N-1} \times \mathbb{R}$ and let $\Sigma=\left\{\left(x^{\prime}, \varphi\left(x^{\prime}\right)\right) ; x^{\prime} \in \mathbb{R}^{N-1}\right\}$. For $P=\left(P^{\prime}, P_{N}\right) \in \Sigma$, we set

$$
\begin{aligned}
& T(P, r)=\left\{\left(x^{\prime}, x_{N}\right) \in \mathbb{R}^{N} ;\left|x^{\prime}-P^{\prime}\right|<r,\left|P_{N}-x_{N}\right|<10 k r\right\} \\
& \omega(P, r)=\left\{\left(x^{\prime}, x_{N}\right) \in T(P, r) ; x_{N}<\varphi\left(x^{\prime}\right)\right\} \\
& A(P, r)=\left(P^{\prime}, P_{N}-5 k r\right) \text { and } \\
& \Sigma(P, r)=\left\{\left(x^{\prime}, x\right) \in \Sigma ;\left|x^{\prime}-P^{\prime}\right|<r\right\} .
\end{aligned}
$$

In the sequel, the dependence on $N$ of the various constants is not made explicit. We note $\delta(x)=d(x, \Sigma)$ for $x=\left(x^{\prime}, x_{N}\right) \in \mathbb{R}^{N}$.

2.2. For $0<\alpha \leq 1$ and $M>0$, we denote $\Lambda(\alpha, M)$ the class of elliptic operators $L$ in $\mathbb{R}^{N}$ in the form

$$
L(u)=\sum_{1 \leq i, j \leq N} a_{i j} \partial_{i j}^{2}(u)+\sum_{1 \leq j \leq N} b_{j} \partial_{j} u+\gamma u
$$


where $a_{i j}, b_{i}$ and $\gamma$ are bounded borel functions on $\mathbb{R}^{N}$ such that when $x, y \in \mathbb{R}^{N}$ and $\xi \in \mathbb{R}^{N}$,

$$
\begin{gathered}
\sum_{i, j} a_{i j}(x) \xi_{i} \xi_{j} \geq M^{-1} \sum_{j} \xi_{j}^{2}, \quad a_{i j}(x)=a_{j i}(x) \\
\left(\sum_{i, j}\left\|a_{i j}\right\|_{\infty}\right)+\left(\sum_{j}\left\|b_{j}\right\|_{\infty}\right)+\|\gamma\|_{\infty} \leq M, \\
\sum_{i, j}\left|a_{i j}(x)-a_{i j}(y)\right| \leq M|x-y|^{\alpha} .
\end{gathered}
$$

2.3. Harnack boundary principle. If $L \in \Lambda(\alpha, M)$, if $P \in \Sigma$, if $u$ and $v$ are two positive $L$-solutions in $\omega(P, r)$ vanishing on $\Sigma(P, r)$ and if $A=A(P, r), r \leq r_{0}$, then

$$
c^{-1} \frac{u(x)}{u(A)} \leq \frac{v(x)}{v(A)} \leq c \frac{u(x)}{u(A)}
$$

for all $x \in \omega(P, r / 2)$, where $c=c\left(k, \alpha, M, r_{0}\right)>0([\mathbf{A 1}]$, see $[\mathbf{A} \mathbf{3}]$ and references there for other related results). More generally, under the same assumptions on $L$ and $u$, if $v$ is positive $L_{1}$-harmonic on $\omega(P, r)$ for some $L_{1} \in \Lambda(\alpha, M)$ having on $\Sigma(P, r)$ the same second order part than $L$, and if $v=0$ on $\Sigma(P, r)$, inequalities (5) hold on $\omega(P, r / 2)$ for some $c=c\left(k, \alpha, M, r_{0}\right)([\mathbf{A} \mathbf{2}])$.

2.4. Ratios of positive harmonic functions near the boundary. The Harnack boundary principle (5) when combined with the maximum principle implies a stronger continuity statement for the ratios of harmonic functions [JK1]. If $u$ and $v$ are positive $L$-solutions on $\omega(P, r)$, $P \in \Sigma, r \leq r_{0}$, vanishing on $\Sigma(P, r)$, and if $A_{\theta}=A(P, r \theta), 0<\theta<1$, then

$$
\left|1-\frac{u(x)}{u\left(A_{\theta}\right)}: \frac{v(x)}{v\left(A_{\theta}\right)}\right| \leq c \theta^{\beta}
$$

for $x \in \omega(P, r \theta / 2)$. Here $c$ and $\beta$ are $>0$ constants (depending only on $k, M, \alpha$ and $\left.r_{0}\right)$.

2.5. Uniform decay property. The following consequence of 2.3 is also needed. There is a constant $\eta=\eta\left(\alpha, M, k, r_{0}\right), 0<\eta \leq 1 / 4$, such that if $u$ is positive $L$-harmonic in $\omega(P, r), P \in \Sigma, r \leq r_{0}$, and $u=0$ on $\Sigma(P, r)$, then $u(x) \leq \frac{1}{2} u(A(P, r))$ for $x \in \omega(P, \eta r)$. It follows that $u(x) \leq C[\delta(x)]^{\gamma} u(A(P, r)), \gamma=\log (2) /|\log (\eta)|$, for some constant $C=C\left(\alpha, M, k, r_{0}\right)$ and $x \in \omega\left(P, \frac{r}{2}\right)$. The opposite estimate, $u(x) \geq$ $C[\delta(x)]^{\gamma^{\prime}} u(A(P, r))$ for $x \in \omega(P, c r)$ and with another constant $\gamma^{\prime}>0$ follows from the local Harnack inequalities. 
2.6. Fatou's Theorem. Denote $\mu_{A}^{\Omega}$ the harmonic measure of $A=$ $A(P, r)$ in $\Omega=\omega(P, r)$ with respect to $L$. If $s$ is positive and $L$-superharmonic in $\Omega$ then $s$ admits a fine limit at $\mu_{A}^{\Omega}$ almost every point $P \in \Sigma(P, r)$, this fine limit being zero $\mu_{A}^{\Omega}$-a.e. if $s$ is a potential. If $s$ is $L$-harmonic in $\Omega$ then $s$ admits a non-tangential limit at $\mu_{A}^{\Omega}$ almost every point $P \in \Sigma(P, r)$. The last property is related to the first by the following fact. If $U \subset \Omega$ is the union of a sequence of balls $B\left(x_{j}, \varepsilon \delta\left(x_{j}\right)\right) \subset \Omega$ where $\varepsilon>0$ is fixed and $x_{j} \rightarrow Q \in \Sigma(P, r)$, then $U$ is not minimally thin (in $\Omega$ ) at $P$ (ref. $[\mathbf{A 1}]$ ).

2.7. Density of harmonic measure. Let $\Omega$ be a domain such that $\Omega \cap T(P, r)=\omega(P, r)$ for some $P \in \Sigma$ and $r \leq r_{0}$ and let $A=A(P, r)$. Let $L \in \Lambda(1, M)$ be formally self-adjoint. The $L$-harmonic measure $\mu_{x}^{\Omega}$ of $x \in \Omega$ is equivalent on $\Sigma(P, r)$ to the natural area-measure $\sigma$. In fact, on $\Sigma^{\prime}=\Sigma(P, r / 2), \mu_{A}^{\Omega}=f_{A} . \sigma$ with $\left\|f_{A}\right\|_{L^{2}\left(\Sigma^{\prime}\right)} \leq C\left\{\sigma\left(\Sigma^{\prime}\right)\right\}^{-\frac{1}{2}}$ where $C=C\left(k, M, r_{0}\right)>0$. This follows from the Rellich formula (see also $[\mathbf{D}],[\mathbf{J K 2}],[\mathbf{A 2} 2])$. Also, $f_{x}>0$ a.e. on $\Sigma^{\prime}$ (the argument of $[\mathbf{D}]$ for $L=\Delta$ is easily extended). The Harnack boundary principle shows that the density $f_{A}$ satisfies also a reverse Hölder inequality. For each $a=\left(a^{\prime}, a_{d}\right) \in \Sigma^{\prime}$ and each positive $t$ with $t<\frac{1}{2} r$ :

$$
\int_{\Sigma(a, t)} f_{A}(x) d \sigma(x) \geq C \sqrt{\sigma(\Sigma(a, t))}\left(\int_{\Sigma(a, t)}\left|f_{A}(x)\right|^{2} d \sigma(x)\right)^{\frac{1}{2}}
$$

where $C=C\left(k, M, r_{0}\right)>0$. By a theorem of Gehring $([\mathbf{G}])$, it follows that $f_{A} \in L^{p}\left(\Sigma^{\prime}\right)$ for some $p=p\left(k, M, r_{0}\right)>2$ with a uniform bound $\left\|f_{A}\right\|_{L_{p}\left(\Sigma^{\prime}\right)} \leq C\left\{\sigma\left(\Sigma^{\prime}\right)\right\}^{\frac{1}{p}-1}, C=C\left(k, M, r_{0}\right)$.

The above extends to wider classes of divergence type elliptic operators (see $[\mathbf{F K P}]$ and references there), and also to every $L$ in $\Lambda(\alpha, M)$, $0<\alpha \leq 1$ ([A4]), but this will not be needed here.

\section{Non-tangential differentiability property}

From now on (Section 3, 4, 5) we consider an operator $L \in \Lambda(1, M)$,

$$
L=\sum_{1 \leq i, j \leq N} a_{i j}(x) \partial_{i} \partial_{j}+\sum_{1 \leq j \leq N} b_{j}(x) \partial_{j}+\gamma
$$

verifying (4) and $\left(4^{\prime}\right)$ with $\alpha=1$. As a first step for the proof of Theorem 1 we prove the next lemma which is probably known but an explicit reference seems difficult to locate (see $[\mathbf{K P}]$ for $L^{p}$ estimates of the non-tangential maximal function of the gradient and a variant of $L^{p}$ convergence, compare also [A2]). We give a proof which relies on Fatou theorem (2.6 above). 
Lemma 1. Let $\Omega$ be a Lipschitz domain in $\mathbb{R}^{N}$. If $u$ is a solution of $L u=0$ in $\Omega$ vanishing on an open subset $S$ of $\partial \Omega$, then $\nabla u$ admits a non-tangential limit at almost every point $P \in S$.

Proof: It is enough to consider the case where $\Omega=\omega(0, r)$ and $S=$ $\Sigma(0, r / 2), r>0$ (with the notations in 2.1 and with respect to some Lipschitz continuous function $\varphi: \mathbb{R}^{N-1} \rightarrow \mathbb{R}$ such that $\left.\varphi(0)=0\right)$. We let $\Omega^{\prime}=\omega(0, r / 2), \Omega^{\prime \prime}=\omega(0,3 r / 4)$ and assume as we may that $u$ is continuous and positive on $\bar{\Omega}$ with $u=0$ on $\Sigma(0, r)$. Then $u \in W_{\text {loc }}^{2, p}(\Omega)$ for all $p<\infty$ ([LU, p. 203-205]) and $u_{\mid \Omega^{\prime \prime}} \in H^{1}\left(\Omega^{\prime \prime}\right)$ (see Remark 1.2 below).

Set $L_{0}=\sum_{1 \leq i, j \leq N} \partial_{i}\left(a_{i j}^{0}(x) \partial_{j}\right)$ where $a_{i j}^{0}(x)=a_{i j}\left(x^{\prime}, \varphi\left(x^{\prime}\right)\right)$ and let $w$ be the solution to the problem $L_{0} w=0$ in $\Omega, w=1$ on $\partial \Omega \backslash \Sigma$, and $w=0$ on $\Sigma$ (compare [A2]). Note that $L_{0} \in \Lambda\left(1, M^{\prime}\right)$ for some $M^{\prime}=M^{\prime}(k, M)>0$ and that $L_{0}$ is self-adjoint. Observe also that $\left(-\partial_{N} w\right)$ is $L_{0}$-harmonic in $\Omega$ (because $L_{0}$ is independent of $\left.x_{N}\right)$ and positive (by the maximum principle). It follows from Harnack inequalities, the uniform decay property 2.5 and the interior gradient estimates that for $x \in \Omega^{\prime \prime}([\mathbf{A} \mathbf{2}])$

$$
0<-\partial_{N} w(x) \leq|\nabla w(x)| \leq c \frac{w(x)}{\delta(x)} \leq-C \partial_{N} w(x) .
$$

By the boundary Harnack principle (5) we have if $x \in \Omega^{\prime \prime}$

$$
|\nabla u(x)| \leq c \frac{u(x)}{\delta(x)} \leq c^{\prime} \frac{w(x)}{\delta(x)} \leq-C \partial_{N} w(x) .
$$

The argument is now broken into three steps. First we note that the distribution $L_{0}\left(\partial_{k} u\right.$ ) (which is defined as an element of $H_{\mathrm{loc}}^{-1}(\Omega)$ since $\left.\partial_{k} u \in H_{\mathrm{loc}}^{1}(\Omega)\right)$ belongs to $H^{-1}\left(\Omega^{\prime}\right)$, i.e. to the dual of $H_{0}^{1}\left(\Omega^{\prime}\right)$. Since $L u=0$,

$$
\begin{aligned}
L_{0}\left(\partial_{k} u\right)=\sum_{1 \leq i, j \leq N} \partial_{k}\left[\left(a_{i j}^{0}-a_{i j}\right) \partial_{i} \partial_{j} u\right]-\partial_{k}\left[\sum_{j=1}^{N} b_{j} \partial_{j} u+\gamma u\right] \\
-\sum_{1 \leq i, j \leq N}\left(\partial_{k} a_{i j}^{0}\right)\left(\partial_{i} \partial_{j} u\right)+\sum_{1 \leq i, j \leq N}\left(\partial_{i} a_{i j}^{0}\right)\left(\partial_{k} \partial_{j} u\right) .
\end{aligned}
$$

Using the Hardy inequality (Remark 1.1) and $\left|a_{i j}(x)-a_{i j}^{0}(x)\right| \leq c \delta(x)$, it is seen that $\left(a_{i j}^{0}-a_{i j}\right) \partial_{j} \partial_{j} u \in L^{2}\left(\Omega^{\prime}\right)$. In fact, on a ball $B=$ 
$B(x, \delta(x) /(40 k)), x \in \Omega^{\prime}$, we have the standard inner estimate ([LU, p. 205])

$$
\int_{B^{\prime}}\left|D^{2} u(z)\right|^{2} d z \leq C \int_{B} \delta(z)^{-4} u(z)^{2} d z
$$

where $\left.B^{\prime}=B(x, \delta(x) /(80 k))\right)$. By a Whitney covering argument, it follows that

$$
\int_{\Omega^{\prime}}\left|\left(a_{i j}-a_{i j}^{0}\right)\left(\partial_{i} \partial_{j} u\right)\right|^{2} d y \leq C \int_{\Omega^{\prime \prime}} \delta^{-2} u^{2} d y<+\infty
$$

This entails that $\sum_{i, j} \partial_{k}\left[\left(a_{i j}^{0}-a_{i j}\right) \partial_{i} \partial_{j} u\right] \in H^{-1}\left(\Omega^{\prime}\right)$. Similarly, using the boundedness of $\partial_{k}\left(a_{i j}^{0}\right)$ it is seen that $\left(\partial_{k} a_{i j}^{0}\right)\left(\partial_{i} \partial_{j} u\right) \in H^{-1}\left(\Omega^{\prime}\right)$. In fact for $v \in H_{0}^{1}\left(\Omega^{\prime}\right)$,

$$
\begin{aligned}
& \int_{\Omega^{\prime}}\left|v \partial_{i} \partial_{j} u\right| d x \leq C\left(\int_{\Omega^{\prime}} \delta^{-2}|v|^{2} \mid d x\right)^{1 / 2}\left(\int_{\Omega^{\prime \prime}} \delta^{-2}|u|^{2} \mid d x\right)^{1 / 2} \\
& \leq C\|\nabla u\|_{L^{2}\left(\Omega^{\prime \prime}\right)}\|\nabla v\|_{L^{2}\left(\Omega^{\prime}\right)}
\end{aligned}
$$

where we have first used that

$$
\int_{B^{\prime} \cap \Omega^{\prime}}\left|v \partial_{i} \partial_{j} u\right| d z \leq C\left[\int_{B^{\prime} \cap \Omega^{\prime}} \delta^{-2} v^{2} d x\right]^{1 / 2}\left[\int_{B} \delta^{-2} u^{2} d x\right]^{1 / 2}
$$

for $x \in \Omega$ as above, and then a Whitney partition, Schwarz and Hardy's inequalities. In the same time we have also shown that $\left(\partial_{i} a_{i j}^{0}\right)\left(\partial_{k} \partial_{j} u\right) \in$ $H^{-1}\left(\Omega^{\prime}\right)$.

Second step: Introduce the function $v \in H_{0}^{1}\left(\Omega^{\prime}\right)$ which is such that $L_{0}(v)=L_{0}\left(\partial_{k} u\right)$ in $\Omega^{\prime}$. Since $v \in H_{0}^{1}\left(\Omega^{\prime}\right)$, a well-known projection argument shows that there is a $L_{0}$-supersolution $p \in H_{0}^{1}\left(\Omega^{\prime}\right)$ such that $|v| \leq p$. By Fatou's theorem (2.6) applied to $p$ and $L_{0}, v$ converges finely (w.r. to $L_{0}$ ) to zero at almost every point $P \in S$. Writing $\partial_{k} u=v+h$, $h$ is a $L_{0}$-solution on $\Omega^{\prime}$, and by (9) we have that $|h| \leq p-C \partial_{N} w$ on $\Omega^{\prime}$. Since $-\partial_{N} w$ is a $>0 L_{0}$-solution in $\Omega$ this means that $|h| \leq-C \partial_{N} w$ and $h$ is hence a difference of two positive $L_{0}$ solutions in $\Omega^{\prime}$. By $2.6, h$ converges finely (and non-tangentially) almost everywhere on $S$. Thus, $\partial_{k} u$ converges finely at almost all point $P \in S$.

Third step: By [LU, p. 205], $\partial_{k} u$ has also the following uniform continuity property: for $x \in \Omega^{\prime}$, and $\left.y \in B(x, \delta(x) / 2)\right)$ one has $\mid \partial_{k} u(y)-$ 
$\partial_{k} u(x)\left|\leq C\|u\|_{\infty, B} \delta^{-1-\alpha}(x)\right| x-\left.y\right|^{\alpha}, B=B\left(x, \frac{3}{4} \delta(x)\right)$, for some constants $\alpha=\alpha(M, r) \in] 0,1]$ and $C>0$. Therefore, by (9) and Harnack inequalities,

$$
\left|\partial_{k} u(y)-\partial_{k} u(x)\right| \leq-C \partial_{N} w(x)\left(\frac{|x-y|}{\delta(x)}\right)^{\alpha} .
$$

It follows that if $P \in S$ is such that $\partial_{N} w$ is non-tangentially bounded at $P$ and $\partial_{k} u$ admits a fine limit $\ell$ at $P$, then $\partial_{k} u$ converges nontangentially to $\ell$ at $P$. If not, a positive number $\varepsilon$ and points $x_{j} \in \Omega^{\prime}$ converging non-tangentially to $P$ could be constructed such that for each $j \geq 1, \inf \left\{\left|\partial_{k} u(x)-\ell\right| ;\left|x-x_{j}\right| \leq \varepsilon \delta\left(x_{j}\right)\right\} \geq \varepsilon$. This means that $\cup_{j \geq 1} B\left(x_{j}, \varepsilon \delta\left(x_{j}\right)\right)$ is thin at $P$, a contradiction (see 2.6 above).

\section{Remarks.}

1.1. Hardy's inequality says that $\int_{\omega^{\prime}} \delta(x)^{-2}|u(x)|^{2} d x \leq c \int_{\omega^{\prime}}|\nabla u(x)|^{2} d x$, where $c=c(k), \omega^{\prime}=\omega\left(P, \frac{r}{2}\right)$, for $u \in H^{1}(\omega(P, r))$ with $u=0$ on $\Sigma(P, r)$. (See $[\mathbf{K K}],[\mathbf{S T E}]$.

1.2. If $u$ is a (continuous) $L$-solution on $\omega(0, r)$ with $u=0$ on $\Sigma(r)$, then $u_{\mid \Omega^{\prime}} \in H^{1}\left(\Omega^{\prime}\right)$ and $\|\nabla u\|_{L^{2}\left(\Omega^{\prime}\right)} \leq c r^{-1}\|u\|_{L^{2}(\Omega)}$ (e.g. extend $u$ by 0 outside $\omega(0, r)$ and apply Lemme 5.2 in $[\mathbf{S}]$ to $u_{+}$and $\left.u_{-}\right)$.

We shall also need the following observation.

Lemma 2. If the function $u$ in Lemma 1 is positive, then $\nabla u(P) \neq 0$ a.e. on $S$.

Proof: We may assume as before that $\Omega=\omega\left(0, r_{0} / 2\right), S=\Sigma\left(0, r_{0} / 4\right)$ and that $u$ vanishes on $\Sigma \cap \partial \Omega$. By the Harnack boundary principle 2.3, we may also assume that $L=L_{0}$ (defined as above) and that $u=w$.

Let $\Omega_{j}=\left\{x \in \Omega ; w(x)>\frac{1}{j}\right\}$ and $S_{j}=\left\{\left(x^{\prime}, x_{N}\right) \in \partial \Omega_{j} \bigcap \Omega ;\left|x^{\prime}\right|<\right.$ $\left.r_{0} / 2\right\}$. By (8) above and for $j$ sufficiently large, $\Omega_{j}$ is of the form $\Omega_{j}=$ $\Omega \cap\left\{\left(x^{\prime}, x_{N}\right) ; x_{N}<\varphi_{j}\left(x^{\prime}\right)\right\}$ where $\varphi_{j}: \mathbb{R}^{N-1} \rightarrow \mathbb{R}$ is $C$-Lipschitz for some constant $C=C\left(M, r_{0}, k\right)$ and of class $C^{1, \alpha}$ for all $\alpha<1$.

On $S_{j}$, the harmonic measure of $A=A\left(0, r_{0} / 2\right)$ w.r. to $L_{0}$ and $\Omega_{j}$ is $\mu_{j}=-\partial_{\nu_{L}}\left(G_{j}(., A)\right) \cdot d \sigma_{S_{j}}$; here $\nu_{L}=\mathcal{A}(\nu)$ on $S_{j}$, where $\nu$ is the exterior unit normal field along $S_{j}$, and $G_{j}$ is the Green's function w.r. to $L_{0}$ in $\Omega_{j}$. This follows from the Stokes formula $\int_{\partial \Omega_{j}}\langle W, \nu\rangle d \sigma=\int_{\Omega_{j}} \operatorname{div}(W) d x$ which is valid for each vector field $W$ of class $W^{1, p}\left(\Omega_{j}\right), p>N$; with $W=\varphi \mathcal{A}\left(\nabla G_{j}(., A)\right)-G_{j}(., A) \mathcal{A}(\nabla \varphi)$, where $\varphi$ is smooth and of support in $T\left(0, r_{0} / 4\right)$ one gets that $\psi(A)=-\int_{S_{j}} \varphi\left\langle\nabla G_{j}(., A), \nu_{L}\right\rangle d \sigma$ for $\psi=$ $\varphi+G_{j}\left(L_{0}(\varphi)\right)$, i.e. $\psi$ is the solution to $L_{0}(\psi)=0$ and $\psi=\varphi$ on $\partial \Omega_{j}$. 
Thus, by the Harnack boundary principle $C^{-1}\left|\partial_{N}(w)\right| \cdot \sigma \leq \mu_{j} \leq$ $C\left|\partial_{N}(w)\right| . \sigma$ on $S_{j}$; in particular $\left\|\partial_{N} w\right\|_{L^{2}\left(S_{j}\right)} \leq C^{\prime}$ for $j$ large. Since the $L_{0}$-harmonic measure $\mu$ of $A$ in $\Omega$ is the weak limit of $\mu_{j}$ and since the Lipschitz constants of the graphs $S_{j}$ are uniformly bounded $C^{\prime \prime-1}\left|\partial_{N}(w)\right| \cdot \sigma \leq \mu \leq C^{\prime \prime}\left|\partial_{N}(w)\right| . \sigma$ on $S$ for some constant $C^{\prime \prime}=$ $C(M, k, r)$. Since the density $f_{A}$ of $\mu$ is $>0$ a.e. on $S$ it follows that $\partial_{N} w \neq 0$ a.e. in $S$.

Remark 2. From 2.3 and the uniform bound $\left\|f_{A}\right\|_{L^{p}(S)} \leq c(k, M, r)$ (with $p=p(M, k)>2, S=\Sigma(0, r / 2)$ ), it follows that every positive $L$-harmonic function $u$ in $\omega(0, r)$ vanishing on $\Sigma(0, r)$ verifies $\|\nabla u\|_{L^{p}(S)} \leq$ $c^{\prime}(k, M, r) u(A(0, r))$. Note also that under the assumptions of Lemma 1 , and if $u \in H^{1}(\Omega)$, a simple limit argument shows that $\nabla u$ coincides on $S$ with the weak gradient $\tilde{\nabla} u \in H_{\text {loc }}^{1 / 2}(S)$ (defined by $\int_{S}\langle\tilde{\nabla} u, \mathcal{A} \nu\rangle d \sigma=$ $\int_{\Omega}\left\{\langle\mathcal{A} \nabla u, \nabla f\rangle+L_{0}(u) f\right\} d x$ for all $f \in H^{1}(\Omega)$ with [supp $\left.\left.f\right] \cap \partial \Omega \subset S\right)$.

\section{The local $C^{0,1}$ approximation}

Recall that we have fixed $L \in \Lambda(1, M)$ with (3), (4), (4') and $\alpha=1$. We set $L_{0}^{\prime}=\sum a_{i j}^{0}(x) \partial_{i} \partial_{j}$ where $a_{i j}^{0}(x)=a_{i j}\left(x^{\prime}, \varphi\left(x^{\prime}\right)\right)$. The operator $L_{0}^{\prime}$ is slightly more convenient now than $L_{0}$ (as defined in Section 3) because its solutions are at least of class $C^{2,1}$. Fix $r_{0}>0$, let $\Omega=\omega\left(0, r_{0}\right), \Omega^{\prime}=\omega\left(0, r_{0} / 2\right)$ (see notations in 1.1) and let $w$ denote now the solution of $L_{0}^{\prime} w=0$ in $\Omega$ such that $w=1$ on $\partial \Omega \backslash \Sigma$ and $w=0$ on $\Sigma\left(0, r_{0}\right)$. We observe that a local $C^{0,1}$ approximation of $\Omega$ at 0 is provided by the level sets $U(w, \varepsilon)=\{w>\varepsilon\} \cap \Omega^{\prime}, \varepsilon>0$. Let $D(r)=\left\{x^{\prime} \in \mathbb{R}^{N-1} ;\left|x^{\prime}\right| \leq r\right\}$.

Lemma 3. For $\varepsilon>0$ small enough, we may write

$$
U(w, \varepsilon)=\left\{\left(x^{\prime}, x_{N}\right) ;\left|x^{\prime}\right|<r_{0} / 2,-5 k \times r_{0}<x_{N}<\varphi_{\varepsilon}\left(x^{\prime}\right)\right\}
$$

where $\varphi_{\varepsilon}: D\left(r_{0} / 2\right) \rightarrow \mathbb{R}$ is of class $C^{2,1}$ and C-Lipschitz for some $C>0$ independent of $\varepsilon$; also $-k \times r_{0}<\varphi_{\varepsilon}\left(x^{\prime}\right)<\varphi(x)$. Moreover, when $\varepsilon$ decreases to zero, $\varphi_{\varepsilon}$ increases to $\varphi$ uniformly on $D\left(r_{0} / 2\right)$, and $\lim _{\varepsilon \rightarrow 0} D \varphi_{\varepsilon}\left(x^{\prime}\right)=D \varphi\left(x^{\prime}\right)$ for almost all $x^{\prime} \in D\left(r_{0} / 2\right)$.

Proof: The first claim follows by the arguments used in the proof of Lemma 2. As before

$$
0<-\partial_{N} w(x) \leq|\nabla w(x)| \leq c \frac{w(x)}{\delta(x)} \leq-C \partial_{N} w(x)
$$


when $x \in \Omega^{\prime}$. Hence if $\varepsilon>0$ is so small that $w(x)>\varepsilon$ for $\left|x^{\prime}\right| \leq r_{0} / 2$ and $x_{N} \leq-k r_{0}$, the implicit function theorem shows that the region $U(w, \varepsilon) \cap \Omega^{\prime}$ is as required by the first claim above. Recall that by Schauder's theory $w$ is locally of class $C^{2,1}$ in $\Omega$. That $\varphi_{\varepsilon} \rightarrow \varphi$ uniformly on $D\left(r_{0} / 2\right)$ is then obvious, since $w$ is continuous on $\Omega \cup \Sigma\left(0, r_{0}\right)$.

The last part of the proposition follows now from Lemma 1, Lemma 2 (applied to $w$ ) and Lemma 4 below. If $\varphi$ is differentiable at $a^{\prime} \in D\left(r_{0} / 2\right)$ and if $\nabla w(x)$ admits a non tangential limit $\alpha$ at $\left(a^{\prime}, \varphi\left(a^{\prime}\right)\right)=a$ with $\alpha=\left(\alpha^{\prime}, \alpha_{N}\right) \neq 0$ (that is $\alpha_{N} \neq 0$ by $\left.(10)\right)$, then

$$
\partial_{j} \varphi\left(a^{\prime}\right)=\lim _{\varepsilon \rightarrow 0} \partial_{j} \varphi_{\varepsilon}\left(a^{\prime}\right)=-\alpha_{j} / \alpha_{N}, \text { for } j=1, \ldots, N-1 .
$$

To see this, fix $\eta>0$ and $j, 1 \leq j \leq N-1$, and apply Lemma 4 below to the function $f(t)=\varphi_{\varepsilon}\left(a^{\prime}+t e_{j}\right)-\varphi\left(a^{\prime}\right)$ with $\beta=\partial_{j} \varphi\left(a^{\prime}\right)$, $u<0<v$ being the closest to zero with $\varphi_{\varepsilon}\left(a^{\prime}+u e_{j}\right)=\varphi(a)+(\beta+\eta) u$, $\varphi_{\varepsilon}\left(a^{\prime}+v e_{j}\right)=\varphi(a)+(\beta-\eta) v$. It follows that for each small enough $\varepsilon>0$, there is a point $x^{\prime}(\varepsilon) \in D\left(r_{0} / 2\right)$ with the following properties:

(a) the $i$-th coordinate $x_{i}^{\prime}(\varepsilon)$ satisfies $x_{i}^{\prime}(\varepsilon)=a_{i}^{\prime}$ if $i \neq j, 1 \leq i \leq N-1$,

(b) $\varphi_{\varepsilon}\left(x^{\prime}(\varepsilon)\right) \leq \varphi(a)+\left(\partial_{j} \varphi\left(a^{\prime}\right) \pm \eta\right)\left(x_{j}^{\prime}(\varepsilon)-a_{j}^{\prime}\right)$,

(c) $\left|\partial_{j} \varphi\left(a^{\prime}\right)-\partial_{j} \varphi_{\varepsilon}\left(x^{\prime}(\varepsilon)\right)\right| \leq \eta$.

Now from (b) it follows that when $\varepsilon \rightarrow 0$ the point $x_{\varepsilon}=\left(x^{\prime}(\varepsilon), \varphi_{\varepsilon}\left(x^{\prime}(\varepsilon)\right)\right)$ converges non tangentially to $a$ in $\Omega$ as well as $a_{\varepsilon}=\left(a^{\prime}, \varphi_{\varepsilon}\left(a^{\prime}\right)\right)$. Hence, since $\nabla w$ has a non tangential limit $\alpha=\left(\alpha^{\prime}, \alpha_{N}\right)$ at $a$ such that $\alpha_{N} \neq 0$,

$$
\begin{aligned}
\partial_{j} \varphi_{\varepsilon}\left(x^{\prime}(\varepsilon)\right) & =-\partial_{j} w\left(x_{\varepsilon}\right) / \partial_{N} w\left(x_{\varepsilon}\right) \\
& =-\partial_{j} w\left(a_{\varepsilon}\right) / \partial_{N} w\left(a_{\varepsilon}\right)+o(1) \\
& =\partial_{j} \varphi_{\varepsilon}\left(a^{\prime}\right)+o(1),
\end{aligned}
$$

and $\lim \sup _{\varepsilon \rightarrow 0}\left|\partial_{j} \varphi_{\varepsilon}\left(a^{\prime}\right)-\partial_{j} \varphi(a)\right| \leq \eta$ by (c).

Thus, $\lim _{\varepsilon \rightarrow 0} \partial_{j} \varphi_{\varepsilon}\left(a^{\prime}\right)=\partial_{j} \varphi(a)=$ n.t. $\lim _{(a, \varphi(a))}\left\{-\partial_{j} w / \partial_{N} w\right\}$ (where n.t. means nontangential).

Lemma 4. Let $f: I \rightarrow \mathbb{R}$ be a function of class $C^{1}$ on some interval $I=[u, v], u<0<v$. Let $\beta \in \mathbb{R}, \eta>0, \psi(t)=\inf \{(\beta+\eta) t,(\beta-\eta) t\}$ and assume that $f(t) \leq \psi(t)$ on $I$, and $f(u)=\psi(u), f(v)=\psi(v)$. Then, there exists $t \in I$ such that $\left|f^{\prime}(t)-\beta\right| \leq \eta$.

Proof: Since $\frac{f(v)-f(u)}{v-u}=\beta-\eta \frac{v+u}{v-u}$ and $\left|\frac{v+u}{v-u}\right| \leq 1$, the lemma follows at once from the mean value theorem. 


\section{The Rellich formula for $u$ in the domain of $L$}

The previous constructions are now used to obtain the following strong $L^{2}$ approximation property. It is well-known that the later implies the desired extension of the Rellich formula. Notations and assumptions are as in the previous section. It is also assumed for sake of simplicity that $\gamma \leq 0$. Recall that $\Omega^{\prime}=\omega_{\varphi}\left(0, \frac{r_{0}}{2}\right)=\omega\left(0, \frac{r_{0}}{2}\right)$ and that $D(r)=\left\{x^{\prime} \in\right.$ $\left.\mathbb{R}^{N-1} ;\left|x^{\prime}\right| \leq r\right\}$.

Theorem 1. Let $\Omega_{j}=\left\{x \in \Omega^{\prime} ; w(x)>\varepsilon_{j}\right\}$, where $\varepsilon_{j} \rightarrow 0, \varepsilon_{j}>0$ and let $\varphi_{j}=\varphi_{\varepsilon_{j}}$. Let $u_{j}, j \geq 1$, be L-harmonic on $\Omega_{j}$ vanishing on $\Sigma_{j}=\left\{w=\varepsilon_{j}\right\}, j \geq 1$. If $u_{j}$ converges uniformly on $\Omega^{\prime}$ to $u$ (set $u_{j}=0$ on $\left.\Omega^{\prime} \backslash \Omega_{j}\right)$, the functions $f_{j}\left(x^{\prime}\right)=\partial_{\nu_{L}} u_{j}\left(x^{\prime}, \varphi_{j}\left(x^{\prime}\right)\right)$ converge strongly in $L^{2}\left(D\left(r_{0} / 4\right)\right)$ to $f\left(x^{\prime}\right)=\partial_{\nu_{L}} u\left(x^{\prime}, \varphi\left(x^{\prime}\right)\right)$ (and in fact in $L^{p}\left(D\left(r_{0} / 4\right)\right.$ ) for some $p=p(k, M)>2$.)

Here, $\partial_{\nu_{L}} u=\langle\mathcal{A} \nabla u, \nu\rangle$ denotes the conormal derivative of $u$ along $\Sigma$ (and $\nu$ is the unit exterior normal), $\partial_{\nu_{L}} u_{j}$ denotes the conormal derivative of $u_{j}$ along $\Sigma_{j}=\left\{\left(x^{\prime}, \varphi_{j}\left(x^{\prime}\right) ; x^{\prime} \in D\left(0, r_{0} / 2\right)\right\}\right.$. Note that if the $u_{j}$ are $\geq 0$, then simple convergence in $\Omega$ already implies uniform convergence on $\omega\left(0, r^{\prime}\right), r^{\prime}<r_{0} / 2$, by boundary Harnack property. Also an obvious decomposition of $u_{j}$ shows that to prove Theorem 1 we may restrict to the case where $u_{j} \geq 0$.

Proof of Theorem 1: Consider first the special case of the sequence $v_{j}=w-\varepsilon_{j}$ with $L=L_{0}^{\prime}$ and denote $f_{j}^{0}, f^{0}$, the corresponding functions $f_{j}$ and $f$. Then by Lemma $1 f_{j}^{0}\left(x^{\prime}\right) \rightarrow f^{0}\left(x^{\prime}\right)=\left\langle\nabla w(x), \nu_{L}(x)\right\rangle$ almost everywhere in $D^{\prime}=D\left(r_{0} / 4\right)$ (where $x=\left(x^{\prime}, \varphi\left(x^{\prime}\right)\right)$ ). Since there is a uniform bound on $\left\|f_{j}^{0}\right\|_{L^{p}\left(D^{\prime}\right)}$ for some $p>2$, it follows that $f_{j}^{0}$ converge (strongly) to $f^{0}$ in $L^{2}\left(D^{\prime}\right)$. And the proposition follows for the case at hand. It is then clear that $g_{j}\left(x^{\prime}\right)=\partial_{\nu_{L}} v_{j}\left(x^{\prime}, \varphi_{j}\left(x^{\prime}\right)\right)$ tends to $f^{0}\left(x^{\prime}\right)$ a.e. in $D^{\prime}$ and in $L^{2}\left(D^{\prime}\right)$ (note that $f_{j}^{0}\left(x^{\prime}\right)=\partial_{\nu_{L_{0}}} v_{j}\left(x^{\prime}, \varphi_{j}\left(x^{\prime}\right)\right)$ ).

In the general case (with $u_{j} \geq 0, u>0$ in $\Omega^{\prime}$ ), consider $h_{j}=f_{j} / f_{j}^{0}$. By Lemma 5 below, this is a sequence of Hölder continuous functions on $\overline{D^{\prime}}$ which is bounded in $C^{\alpha}\left(\overline{D^{\prime}}\right)$ for some $\alpha, 0<\alpha<1$. Moreover the function $h=f / f^{0}$ - which may be seen as a Hölder continuous function on $\overline{D^{\prime}}$ - is the unique cluster value of this sequence in $L^{\infty}\left(D^{\prime}\right)$. In fact, by Lemma 5 , if $H$ is such a cluster value and if $\eta>0$ is small, both quantities

$$
\left|1-\left[H\left(x^{\prime}\right):(u(A) / w(A))\right]\right| \text { and }\left|1-\left[\left(f\left(x^{\prime}\right) / f^{0}\left(x^{\prime}\right)\right):(u(A) / w(A))\right]\right|,
$$

where $x^{\prime} \in D^{\prime}$ and $A=\left(x^{\prime}, \varphi\left(x^{\prime}\right)-\eta\right)$, are bounded by $\leq c \eta^{\delta}$ for some positive real $\delta$. 
Thus, $h_{j} \rightarrow H$ in $L^{\infty}\left(D^{\prime}\right)$. Since $f_{j}^{0} \rightarrow f^{0}$ almost everywhere on $D^{\prime}$ and in $L^{2}\left(D^{\prime}\right)$, it follows that $f_{j} \rightarrow f$ in $L^{2}\left(D^{\prime}\right)$ and almost everywhere on $D^{\prime}$ which proves the theorem.

Recall that for $a=\left(a^{\prime}, a_{N}\right) \in \Sigma, T(a, \eta)=\left\{\left(x^{\prime}, x_{N}\right) ;\left|x^{\prime}-a^{\prime}\right|<\right.$ $\left.\eta,\left|x_{N}-a_{N}\right|<10 k \eta\right\}$. Let $S=\Sigma \cap\left\{\left(x^{\prime}, \varphi\left(x^{\prime}\right)\right) ;\left|x^{\prime}\right|<r_{0} / 4\right\}$ and $U_{\varepsilon}=\omega\left(0, r_{0} / 2\right) \bigcap\{w>\varepsilon\}$. Set $w_{\varepsilon}=w-\varepsilon$ for each $\varepsilon>0$.

Lemma 5. There are constants $C>1$ and $D \in(0,1)$ with the following property. If $P \in S$, if $h$ is positive L-harmonic in $T(P, \eta) \cap U_{\varepsilon}$ vanishing on $T_{P}(\eta) \cap \partial U_{\varepsilon}, \varepsilon>0$, and if $\eta>0$ is small, then

$$
\left(1-C \eta^{D}\right) \frac{w_{\varepsilon}(x)}{w_{\varepsilon}\left(A_{\eta}^{\prime}\right)} \leq \frac{h(x)}{h\left(A_{\eta}^{\prime}\right)} \leq\left(1+C \eta^{D}\right) \frac{w_{\varepsilon}(x)}{w_{\varepsilon}\left(A_{\eta}^{\prime}\right)}
$$

for $x \in T\left(P, \frac{\eta^{2}}{4 C}\right) \cap U_{\varepsilon}$ and $A_{\eta}^{\prime}=\left(P^{\prime}, P_{N}-5 k \eta^{2}\right)$.

Note that if $A_{\eta} \notin U_{\varepsilon}$, then $T\left(P, \frac{\eta^{2}}{4 C}\right) \cap U_{\varepsilon}=\emptyset$ at least if $\eta$ is small.

Proof: We use a construction from [A 2] . Let $s=f(w)$ where $f(t)=$ $\int_{0}^{t} e^{-\theta^{\alpha}} d \theta, u=g(w)$ where $g(t)=\int_{0}^{t} e^{\theta^{\alpha}} d \theta$ and $0<\alpha<1$. It is immediately checked, using (9), that if $\alpha$ is small, then $s$ (resp. $u$ ) is $L$-superharmonic (resp. $L$-subharmonic) in $\Omega^{\prime} \bigcap\{w<\varepsilon\}$ for $\varepsilon>0$ small. In fact,

$$
L(s)=f^{\prime \prime}(w)\left\{\sum a_{i j} \partial_{i} w \partial_{j} w\right\}+f^{\prime}(w) L(w)+\gamma\left(f(w)-w f^{\prime}(w)\right)
$$

so that using (9) and Schauder interior estimates, we have on $\Omega^{\prime}$ near $\Sigma$,

$$
\begin{aligned}
\left\{\sum a_{i j} \partial_{i} w \partial_{j} w\right\}^{-1} L(s) \leq & f^{\prime \prime}(w)+C|\nabla w|^{-2} f^{\prime}(w)\left\{L(w)-L_{0}^{\prime}(w)\right\} \\
& +C|\nabla w|^{-2} w \\
\leq & f^{\prime \prime}(w)+C^{\prime} \frac{\delta^{2}}{w^{2}} f^{\prime}(w)\left(\delta \frac{w}{\delta^{2}}+\frac{w}{\delta}+w\right)+C^{\prime} \frac{\delta^{2}}{w} \\
\leq & f^{\prime \prime}(w)+C^{\prime \prime} w^{\beta-1}\left(f^{\prime}(w)+1\right)
\end{aligned}
$$

for some positive constants $C^{\prime \prime}$ and $\beta$, and where in the last line we have used 2.5. It follows that if we fix $\alpha$ in $(0, \beta)$, then $s$ is $L$-superharmonic near $\Sigma$ in $\Omega^{\prime}$. The subharmonicity of $u=g(w)$ is obtained similarly.

Let $s_{\varepsilon}=s-f(\varepsilon)=f(w)-f(\varepsilon)$ and $u_{\varepsilon}=u-g(\varepsilon)=g(w)-g(\varepsilon)$ for $\varepsilon>0$. If $m=\sup \left\{w(x) ; x \in T(P, \eta) \cap U_{\varepsilon}\right\}, P \in S$,

$$
e^{-m^{\alpha}} \leq s_{\varepsilon}(x) / w_{\varepsilon}(x) \leq e^{-\varepsilon^{\alpha}}
$$


when $x \in \omega=U_{\varepsilon} \cap T(P, \eta)$. Similarly, we have $e^{\varepsilon^{\alpha}} \leq u_{\varepsilon}(x) / w_{\varepsilon}(x) \leq e^{m^{\alpha}}$ for $x \in \omega$. Observe also that

$$
e^{-\left(\varepsilon^{\alpha}-m^{\alpha}\right)} \leq e^{(m-\varepsilon)^{\alpha}} \leq \exp \left(c^{\prime} \eta^{b \alpha}\right)=e^{c^{\prime} \eta^{\beta^{\prime}}}
$$

for some constants $b>0$ and $c^{\prime}>0$, where we have applied again 2.5.

Now, let $\tilde{s}_{\varepsilon}=\frac{m-\varepsilon}{(f(m)-f(\varepsilon))} s_{\varepsilon}$ and $\tilde{u}_{\varepsilon}=\frac{m-\varepsilon}{(g(m)-g(\varepsilon))} u_{\varepsilon}$. For $\eta>0$ and small, the function $\tilde{s}_{\varepsilon}$ (resp. $\tilde{u}_{\varepsilon}$ ) is positive $L$-superharmonic (resp. $L$ subharmonic) on $\omega=T(P, \eta) \cap U(\varepsilon)$, vanishes on $\Sigma_{\varepsilon}=\partial U_{\varepsilon} \cap T(P, \eta)$ and $\tilde{u}_{\varepsilon} \leq \tilde{s}_{\varepsilon}$ in $\omega$. Taking the smallest $L$-harmonic majorant of $\tilde{u}_{\varepsilon}$ in $\omega$, we obtain a positive $L$-harmonic function $h_{1}$ on $\omega$ such that $\tilde{u}_{\varepsilon} \leq h_{1} \leq \tilde{s}_{\varepsilon}$ on $\omega$. Of course, $h_{1}$ vanishes on $\Sigma_{\varepsilon}$ and by the previous estimates, we have in $\omega$

$$
\left(1-c \eta^{\beta^{\prime}}\right) w_{\varepsilon}(x) \leq h_{1}(x) \leq\left(1+c \eta^{\beta^{\prime}}\right) w_{\varepsilon}(x)
$$

Finally, if $h$ is any positive $L$-harmonic function on $\omega$ vanishing on $\Sigma_{\varepsilon}$, we know (see Section 2.4) that for some real $\beta^{\prime \prime} \in(0,1]$

$$
\left(1-c \eta^{\beta^{\prime \prime}}\right) \frac{h_{1}(x)}{h_{1}\left(A_{\eta}^{\prime}\right)} \leq \frac{h(x)}{h\left(A_{\eta}^{\prime}\right)} \leq\left(1+c \eta^{\beta^{\prime \prime}}\right) \frac{h_{1}(x)}{h_{1}\left(A_{\eta}^{\prime}\right)}
$$

when $x \in U_{\varepsilon} \cap T\left(P, \frac{\eta^{2}}{4 C}\right)$. Combining (12) and (13) we obtain (11).

Let now $L \in \Lambda(1, M)$ be in the form $L=\sum \partial_{i}\left(a_{i j} \partial_{j}\right.$.) the $a_{i j}$ satisfying (4) and $\left(4^{\prime}\right)$ with $\alpha=1$. From Theorem 1 , the desired generalization of Rellich formula (1) together with an extension of Theorem 1 itself are easily derived. In the next corollary notations are the same as in Theorem 1.

Corollary 1. Let $v \in H_{0}^{1}\left(\Omega^{\prime}\right)$ be such $L(v)=f \in L^{2}\left(\Omega^{\prime}\right)$, and let $v_{j} \in H_{0}^{1}\left(\Omega_{j}^{\prime}\right)$ be such that $L\left(v_{j}\right)=f$ in $\Omega_{j}^{\prime}$. Then, $\partial_{\nu_{L}} v_{j}\left(x^{\prime}, \varphi_{j}\left(x^{\prime}\right)\right) \rightarrow$ $\partial_{\nu_{L}} v\left(x^{\prime}, \varphi\left(x^{\prime}\right)\right)$ in $L^{2}\left(D^{\prime}\right)$.

Recall (ref. $[\mathbf{N}]$ ) that if $W$ be a bounded Lipschitz region in $\mathbb{R}^{N}$, then for $u \in H_{0}^{1}(W)$ such that $L(u)=f \in L^{2}(W)$, the weak conormal derivative $\partial_{\nu_{L}}(u)$ (defined as a member of $H^{-\frac{1}{2}}(\partial W)$ ) belongs to $L^{2}(\partial \Omega)$ and $\left\|\partial_{\nu_{L}}(u)\right\|_{L^{2}(\partial W)} \leq C(W, M)\|f\|_{L^{2}(\partial W)}$. This follows from a natural approximation argument combined with Rellich formula for functions in $H^{2}$. By Theorem 1 , if $f=0$ in an open neighborhood $V$ of $P \in \partial W$, then the weak and the strong conormal derivatives of $v$ coincide in $V \cap \partial W$. 
Proof of Corollary 1: By decomposing $f_{j}$ into its positive and negative parts we may assume that $f_{j} \leq 0$ in $\Omega_{j}^{\prime}, j \geq 1$. By Rellich formula there is a uniform estimate $\left\|\partial_{\nu_{L}}(w)\right\|_{L^{2}\left(\Omega_{j}^{\prime}\right)} \leq C\|f\|_{L^{2}\left(\partial \Omega_{j}^{\prime}\right)}$ for $w \in H_{0}^{1}\left(\Omega_{j}^{\prime}\right)$ with $L(w)=f \in L^{2}\left(\Omega_{j}^{\prime}\right)$ and a constant $C$ independent of $j$. Thus by a standard approximation argument we may also assume that $f_{j}=0$ on a neighborhood $V$ of $\Sigma$. Then, $v_{j} \rightarrow v$ simply on $V \cap \Omega^{\prime}$ and the result follows from Theorem 1 .

\section{Remarks.}

5.1. It follows from Lemma 1 and Theorem 1 (and the obvious approximation argument) that in a given bounded region $\Omega$ the $L$-harmonic measure of $x_{0} \in \Omega$ induces on a Lipschitz open piece $S$ of $\partial \Omega$ the measure of density $-\partial_{\nu_{L}}\left(G\left(x_{0},.\right)\right.$, if $G$ denotes the Green's function of $L$ in $\Omega$.

5.2. Corollary 1 is easily extended to the following case: $v \in H^{1}\left(\Omega^{\prime}\right)$ with $L(v)=f \in L^{2}\left(\Omega^{\prime}\right)$ in $\Omega^{\prime}$ and $v=F$ on $\partial \Omega$ for some $F \in H^{2}\left(\Omega^{\prime}\right)$; the $v_{j} \in H^{1}\left(\Omega_{j}^{\prime}\right)$ are such that $L\left(v_{j}\right)=f$ in $\Omega_{j}^{\prime}$ and $v_{j}=F$ in $\partial \Omega_{j}^{\prime}$. One has just to look to $v_{j}^{\prime}=v_{j}-F$ and to notice that $\nabla F\left(x^{\prime}, \varphi_{j}\left(x^{\prime}\right)\right) \rightarrow$ $\nabla F\left(x^{\prime}, \varphi\left(x^{\prime}\right)\right)$ a.s. in $D\left(r_{0} / 4\right)$ and also in $L^{2}\left(D^{\prime}\right)$ (in fact in $H^{1 / 2}\left(D^{\prime}\right)$ ) since $\nabla F \in H^{1}(\Omega)$.

From Corollary 1 and Remark 5.2 above the extension of Rellich formula follows.

Corollary 2. Let $L$ be as before, let $V$ be a $C_{0}^{1}$ vector field in $\mathbb{R}^{N}$ and let $\Omega$ be a domain in $\mathbb{R}^{N}$ which is Lipschitz in a neighborhood of each point $P$ of $F=\operatorname{supp}(V) \bigcap \partial \Omega$. If $u \in H^{1}(\Omega)$ is such that $L(u) \in L^{2}(\Omega)$ and if $u=g$ in a neighborhood of $F$ in $\partial \Omega$ for some $g \in H^{2}(\Omega)$ then the Rellich formula (1) holds.

To state the next corollary, we assume that we are given a sequence of functions $\psi_{j}$ in $D\left(r_{0}\right)$ such that $\psi_{j} \leq \varphi,\left|\psi_{j}(x)-\psi_{j}(y)\right| \leq k|x-y|$ for $x, y$ in $D\left(r_{0}\right), \lim _{j \rightarrow \infty}\left\|\psi_{j}-\varphi\right\|_{\infty}=0$ and $\lim _{j \rightarrow \infty} D \psi_{j}\left(\overline{x^{\prime}}\right)=D \varphi\left(x^{\prime}\right)$ for almost all $x^{\prime} \in D\left(r_{0}\right)$. We let $\Omega^{\prime}=\omega_{\varphi}\left(0, r_{0} / 2\right)$ (as before) and $\Omega_{j}=\Omega^{\prime} \cap\left\{\left(x^{\prime}, x_{N}\right) ; x_{N}<\psi_{j}\left(x^{\prime}\right)\right\}$. Set $\Sigma_{j}=\left\{\left(x^{\prime}, \psi_{j}\left(x^{\prime}\right)\right) ; x^{\prime} \in D\left(r_{0}\right)\right\}$, $\Sigma=\left\{\left(x^{\prime}, \varphi\left(x^{\prime}\right)\right) ; x^{\prime} \in D^{\prime}\left(r_{0}\right)\right\}$, and $\nu_{j}$ (resp. $\nu$ ) to denote the exterior unit normal field on $\Sigma_{j}$ (resp. on $\Sigma$ ).

Corollary 3. Let $L$ be as in Corollary 1 and let $\left\{u_{j}\right\}$ be a sequence of functions such that $u_{j} \in H^{1}\left(\Omega_{j}\right), u_{j}=0$ on $\Sigma_{j}$ and $L\left(u_{j}\right)=f_{j} \in$ $L^{2}\left(\Omega_{j}\right)$. Assume that $f_{j} \rightarrow f$ in $L^{2}\left(\Omega^{\prime}\right)$ (set $f_{j}=0$ in $\Omega_{j}^{c}$ ) and that 
$u_{j} \rightarrow u$ in $H_{\mathrm{loc}}^{1}\left(\Omega^{\prime}\right)$. Then, $u \in H^{1}\left(\omega_{\varphi}\left(0, \frac{3 r}{8}\right)\right), u=0$ in $\Sigma\left(r_{0} / 4\right)$, and $\partial_{\nu_{j}} u_{j}\left(x^{\prime}, \psi_{j}\left(x^{\prime}\right)\right) \rightarrow \partial_{\nu} u\left(x^{\prime}, \varphi\left(x^{\prime}\right)\right)$ in $L^{2}\left(D\left(r_{0} / 4\right)\right)$.

This follows from Rellich formula (Corollary 2) and the fact that in a Hilbert space $(H,\|\|$.$) every weakly convergent sequence \theta_{j}$ such that $\lim _{j \rightarrow \infty}\left\|\theta_{j}\right\|=\left\|\lim _{j \rightarrow \infty} \theta_{j}\right\|$ is strongly convergent. Corollary 3 means that for a bounded Lipschitz domain $\Omega$ with a given $C^{0,1}$ approximation (ref. $[\mathbf{N}]$ ) by a sequence of Lipschitz domains $\Omega_{j}$ the following holds: if $v_{j} \in H_{0}^{1}\left(\Omega_{j}\right), v \in H_{0}^{1}(\Omega)$ are such $L\left(v_{j}\right)=f_{j} \in L^{2}\left(\Omega_{j}\right)$ converges strongly (in the appropriate sense) to $L(v)=f \in L^{2}(\Omega)$, then $\nabla v_{j \mid \partial \Omega_{j}} \rightarrow$ $\nabla v_{\mid \partial \Omega}$ in the appropriate strong $L^{2}$ sense.

\section{References}

[A1] A. Ancona, Principe de Harnack à la frontière et théorème de Fatou pour un opérateur elliptique dans un domaine lipschitzien, Ann. Inst. Fourier 28(4) (1978), 169-213.

[A2] A. Ancona, Comparaison des fonctions de Green et des mesures harmoniques pour des opérateurs elliptiques, C. R. Acad. Sci. Paris 294 (1982), 505-508.

[A3] A. AnCOnA, Negatively curved manifolds, elliptic operators and the Martin boundary, Ann. of Math. 125 (1987), 495-536.

[A4] A. Ancona, First eigenvalues and comparison of Green's functions for elliptic operators on manifolds and domains, J. Anal. Math. 72 (1997), 45-92.

[C] A. ChaїrA, Controlabilité de l'équation des ondes, Manuscript (1995).

[CL] A. Chaїra and G. Lebeau, Private conversations.

[CFMS] L. Cafarelli, E. Fabes, S. Mortola and S. Salsa, Boundary behavior of non-negative solutions of elliptic operators in divergence form, Indiana Univ. Math. J. 30 (1981), 621-640.

[D] B. E. J. DAhlberG, On estimates of harmonic measure, Arch. Rational Mech. Anal. 1965 (1977), 272-288.

[FKP] R. A. Fefferman, C. Kenig and J. Pipher, The theory of weight and the Dirichlet problem for elliptic equations, Ann. of Math. 134 (1991), 65-124.

[JK1] D. Jerison And C. Kenig, Positive harmonic functions in non tangentially accessible domains, Adv. Math. 46 (1982), 80-147.

[JK2] D. JERISON AND C. Kenig, The Dirichlet problem in non smooth domains, Ann. of Math. 113 (1981), 367-382. 
[KK] J. KADLEC AND A. KufNeR, Characterization of functions with zero traces by integrals with weight functions, Časopis Pěst. Mat. 91 (1966), 91-113.

[KP2] C. KEnIG AND J. PIPHER, Neumann problem for elliptic equations with non-smooth coefficients, Invent. Math. 113 (1993), 447509.

[LU] O. A. Ladyzhenskaia And N. N. URaltseva, "Linear and quasi-linear equations," Academic Press, New York and London, 1968.

[N] J. NEČAs, "Les méthodes directes en théorie des opérateurs elliptiques," Masson\&co (Paris), Academia (Prague), 1967.

[S] G. Stampacchia, Le problème de Dirichlet pour les équations elliptiques du second ordre à coefficients discontinus, Ann. Inst. Fourier 15(1) (1965), 189-258.

[Ste] E. M. SteIn, "Singular integrals and differentiability properties of functions," Princeton University Press, Princeton New Jersey, 1970.

Département de Mathématiques

Université Paris Sud

Campus d'Orsay, Bât. 425

Orsay 91405

FRANCE

e-mail: ancona@matups.math.u-psud.fr

Rebut el 2 de setembre de 1997 